\title{
Robert Cénalis et l'histoire épiscopale d'Avranches de 1100 à 1253
}

\author{
RICHARD ALLEN*
}

\begin{abstract}
T 'histoire De L'ANCien Diocèse d'Avranches, le plus petit de la province ecclésiastique de Rouen, est marquée par une suite de malheurs. Complètement coupé du reste de l'Église normande après les incursions scandinaves du $\mathrm{IX}^{\mathrm{e}}$ siècle ${ }^{1}$, les évêques $\mathrm{du} \mathrm{XI}^{\mathrm{e}}$ et $\mathrm{du} \mathrm{XII}{ }^{\mathrm{e}}$ siècle s'efforcèrent de reconstituer la richesse de leur cathédrale ${ }^{2}$, mais elle fut à nouveau dévastée par les Bretons au XIII ${ }^{\mathrm{e}}$ siècle, par les Anglais au XV siècle, et par les protestants en $1562^{3}$. Une autre catastrophe, d'une nature très différente, s'est abattue, il y a deux siècles, sur la cathédrale d'Avranches : déjà partiellement ruinée avant la Révolution française, elle disparut complètement au début du XIX ${ }^{\mathrm{e}}$ siècle, et on ne la connaît aujourd'hui que par des dessins ${ }^{4}$, des plans, et par les résultats des fouilles menées dans les années $1970^{5}$. La perte de ce monument historique est compensée par la survie des quelques manuscrits appartenant à la bibliothèque médiévale de la cathédrale, dont l'un est un cartulaire ${ }^{6}$. Mais les destructions causées par la Révolution au XVIII ${ }^{\mathrm{e}}$ siècle, et celles infligées aux archives de Saint-Lô par les bombardiers alliés en $1944^{7}$, représentent un véritable traumatisme pour le patrimoine écrit médiéval de la région, et en particulier ce qui concerne les évêques d'Avranches ${ }^{8}$.
\end{abstract}

* St John's College, Université d'Oxford.

1 La destruction de la région était si profonde que le siège resta vacant pendant plus d'un siècle, Gallia christiana in provincias ecclesiasticas distributa, Paris, 16 vol., 1865-1870, t. XI, col. 474.

2 R. Allen, «Un évêque et sa ville : les évêques d'Avranches de 990 à 1134 », Revue de l'Avranchin et du pays de Granville, 86, 2009, p. 1-49.

3 Pour les pillages du XIII ${ }^{\mathrm{e}}$ siècle, voir ci-dessous. Pour ceux des $\mathrm{XV}^{\mathrm{e}}$ et $\mathrm{XVI}^{\mathrm{e}}$ siècles, voir D. Levalet, "La cathédrale Saint-André et les origines chrétiennes d'Avranches », Archéologie médiévale, 12, 1982, p. 123 ; F. Gueroult, " Procès-verbal du pillage de la cathédrale et de l'Évêché d'Avranches par les Protestants, en 1562 ", Revue de l'Avranchin, 9, 1898, p. 147-171; Bibl. mun. Avranches, fonds Pigeon, ms. 45, p. 497.

4 M. BAYLÉ, «Les évêques et l'architecture normande au XI ${ }^{\mathrm{e}}$ siècle », dans P. Bouet et F. Neveux (dir.), Les évêques normands du XI siècle, Caen, 1995, p. 159.

5 D. Levalet, «La cathédrale Saint-André... », op. cit., p. 107-153.

6 Bibl. mun. Avranches, ms. 206.

7 Y. NÉDÉLEC, « Répertoire des bibliothèques et archives de la Manche », Revue du département de la Manche, 4, 1962, p. 394-395.

8 Grâce aux efforts de M. Natalis de Wailly, ancien directeur de l'École des chartes, plus de 1500 , chartes des abbayes de Savigny et de Mortain ont été transférées en 1839 de la sous-préfecture de Mortain aux Archives nationales (Arch. nat., L 966 à 979). Parmi ces pièces emportées à Paris figurent 61 chartes des évêques d'Avranches de 1134 jusqu'en 1253. L'auteur est en train d'éditer ces chartes en vue d'une histoire consacrée aux actes des évêques d'Avranches. 
Les spécialistes conscients de ces problèmes ont récemment commencé à apporter d'importants éclaircissements en étudiant les copies faites par des personnages tels que le chanoine Charles Guérin (1624-1711)9 ${ }^{9}$ dont les transcriptions se trouvent aujourd'hui parmi les manuscrits de la Bibliothèque municipale d'Avranches ${ }^{10}$. Ce chanoine s'appuya sur quelques manuscrits actuellement perdus, y compris un inventaire de l'évêché rédigé par l'évêque Laurent de Faye (1379-1391) et un cartulaire du Xv siècle connu sous le nom de Livre Blanc. Les copies de Guérin furent d'abord mises au jour vers la fin du XIX ${ }^{\mathrm{e}}$ siècle par l'abbé Pigeon (1829-1902) ${ }^{11}$, qui tira également des renseignements d'un autre manuscrit méconnu conservé dans les fonds latins de la Bibliothèque nationale. Ce manuscrit, provenant de la collection du bibliophile Louis-Émery Bigot (1626-1689), est intitulé Hierarchia Neustrice ${ }^{12}$, et fut écrit par Robert Cénalis, évêque d'Avranches (1532-1560). Le manuscrit lui-même est divisé en cinq tomes, dont les deux premiers contiennent des renseignements sur l'histoire épiscopale avranchaise ${ }^{13}$. C'est le second de ces deux tomes qui nous intéresse, et en particulier les parties traitant des évêques du XII ${ }^{\mathrm{e}}$ et du XIII ${ }^{\mathrm{e}}$ siècle, qui contiennent de précieux renseignements, non seulement pour l'histoire d'Avranches, mais également pour celle du duché normand.

Dix pontifes ont gouverné l'église avranchinaise de 1100 jusqu'en $1253^{14}$, et pour chacun d'entre eux le manuscrit Cénalis donne une courte notice bibliographique. Ces notes indiquent dans un premier temps des détails sur les funérailles de l'évêque, puis développent ensuite les faits de son épiscopat. Les événements extérieurs au diocèse, qui fournissent souvent des références chro-

9 Sur Charles Guérin, voir la courte notice biographique qui figure dans les annales d'Avranches dites de 1648 ("Chronique d'Avranches aux XviI et XviII ${ }^{\mathrm{e}}$ siècles ", E.-A. Pigeon (éd.), Mémoires de la Société d'archéologie d'Avranches, 7, 1885, p. 220-224). Ce Guérin ne doit pas être confondu avec un autre Charles Guérin, qui vivait au XIX ${ }^{\mathrm{e}}$ siècle et qui a aussi laissé des copies de documents (v. D. Perrin, « Chalandrey dans les registres paroissiaux du Mesnil-Thibault ", Revue de l'Avranchin et du pays de Granville, 72, 1995, p. 201-242 et 281-306), ni avec le docteur Louis-Julien Guérin, mort en 1843, qui a arraché le crâne de saint Aubert des mains des révolutionnaires.

10 Bibl. mun. Avranches, fonds Pigeon, ms. 45. Pour les éditions des documents trouvés dans ce manuscrit, voir J. Peltzer, « Portchester, les évêques d'Avranches et les Hommet (1100-1230) », Annales de Normandie, 56, 2006, p. 463-482 ; R. Allen, "Un évêque et sa ville... », op. cit., p. 1-49.

11 É.-A. Pigeon, Le diocèse d'Avranches, sa topographie, ses origines, ses évếques, sa cathédrale, ses églises, ses comtes et ses châteaux, Coutances, 2 vol., 1888, t. II, p. 658-678.

$12 \mathrm{BnF}, \mathrm{ms}$. lat. 5201. Le titre complet est « Hierarchia Neustria authore reverendo in Christo patre Roberto Cenali divina clementia episcopo Arboricensi theologo doctore ordine et origine Parisiensi in quinque secreta tomos".

$13 \mathrm{BnF}$, ms. lat. 5201, fol. 1r-91v. Les autres tomes traitent des archevêques de Rouen (fol. 92r-132r), des abbés du Mont-Saint-Michel (fol. 135r-149v) et des abbés de la Normandie (fol. 150r-168v).

14 D. SPEAR, The personnel of the Norman cathedrals during the ducal period, 911-1204, Londres, 2006, p. 4-6 ; J. Peltzer, Canon law, careers and conquest : episcopal elections in Normandy and Greater Anjou, c. 1140-c. 1230, Cambridge, 2008, p. 150-166. Un onzième évêque, Guillaume de Chemillé (11961197/1199), fut élu mais jamais consacré. 
nologiques, sont aussi considérés, tandis que l'auteur s'appuie sur des sources variées. Ces sources comprennent les documents perdus autrefois conservés aux archives diocésaines, comme l'inventaire et le cartulaire cités ci-dessus, ainsi que des copies des textes répandus telles que les lettres d'Arnoul, évêque de Lisieux (1141-1181), la chronique de Robert de Torigni (1154-1186), l'Historia ecclesiastica d'Orderic Vital ${ }^{15}$. Ce sont naturellement les textes tirés des archives avranchaises qui nous intéressent, en particulier parce que ces documents ont largement dépassé le cadre de la communauté des médiévistes.

Ceci est particulièrement vrai pour ce qui concerne la dédicace de l'église d'Avranches, qui fut parmi les dernières des cathédrales normandes consacrées pendant l'époque ducale ${ }^{16}$. Bien que la date de cet événement soit bien connue (17 septembre 1121) ${ }^{17}$, la source de cette information n'a jamais été précisée. Mais une note marginale du manuscrit Cénalis nous informe sur la manière dont la mention de la dédicace put être trouvée dans un cartulaire, ainsi que dans d'autres " textes anciens ${ }^{18}$. Étant donné que le cartulaire appelé Livre Vert mentionne seulement la dédicace sans détail ${ }^{19}$, cette note laisse à penser que l'information sur la dédicace provient du cartulaire perdu, le Livre Blanc. Quant aux textes anciens, il est possible que Robert Cénalis ait consulté les chartes originales, aujourd'hui perdues. Malheureusement, cette note marginale ne donne aucun autre détail sur la dédicace, qui selon un manuscrit du XVII ${ }^{\mathrm{e}}$ siècle eut lieu en présence du roi-duc Henri I ${ }^{\text {er }}$, nommé par erreur Henri II, et des évêques de Bayeux, de Coutances, de Sées et du Mans ${ }^{20}$. La raison du choix de cette date reste également inconnue. La cathédrale fut consacrée en l'honneur de saint André, dont la fête est le 30 novembre. Cela dit, d'autres évêques nor-

15 BnF, ms. 1at. 5201, fol. 65v-68v, 71r-75v.

16 La dédicace de la cathédrale de Rouen eut lieu en 1063 (R. Allen, "The Acta archiepiscoporum Rotomagensium: study and edition ", Tabularia «Documents", 9, 2009, p. 40); celle de Bayeux le 14 juillet 1077 (J.-M. Bouvris, « La dédicace de l'église cathédrale Notre-Dame de Bayeux (14 juillet 1077) ", Société des sciences, arts et belles-lettres de Bayeux, 28, 1982, p. 3-16); celle de Coutances le 8 décembre 1056 (Recueil des actes des ducs de Normandie de 911 à 1066, M. Fauroux (éd.), Caen, 1961, nº 214, p. 407); celle d'Évreux en 1077 (R. Allen, The Norman episcopate, 989-1110, thèse doctorale, University of Glasgow, 2 vol., 2009, t. I, p. 229-230), et celle de Lisieux le 8 juillet ca. $1060 \times 1070$ (R. Allen, The Norman episcopate ..., op. cit., t. I, p. 265). La cathédrale de Sées fut reconsacrée le 2 janvier 1049 après un incendie, mais peu après ses murs vont s'effondrer (The Gesta Normannorum ducum of William of Jumièges, Orderic Vitalis, and Robert of Torigni, E.M.C. van Houts (éd.), Oxford, 2 vol., 1992-1995, II, p. 114-116). Le nouvel édifice n'est consacré que le 21 mars 1126 (The Ecclesiastical History of Orderic Vitalis, M. CHI BNALL (éd.), Oxford, 6 vol., 1969-1980, t. VI, p. 366).

17 Voir, par exemple, Gallia christiana, t. XI, cols. 466-467.

18 "Ecclesia siquidem Abrincensis (ubi ex chartulario colligitur) fuit dedicata et consecrata in nomine Domini, et beatissimi Andrea ipsius apostoli, fratris Simonis Petri, prout reperitur in antiquis scriptis de anno Domini millesimo centesimo vigesimo primo, calendarum octob(ris) 15, qua die in eadem ecclesia celebratur annuatim solemniter festum dictae dedicationis cum octavis solemnibus », BnF, ms. lat. 5201, fol. 65v (en marge).

19 Bibl. mun. Avranches, ms. 206, fol. 8r.

20 N.-J. Foucault, «Eslection d'Avranches ", dans L'intendance de Caen en 1700 : édition critique des mémoires rédigés sous la dir. de Nicolas-Joseph Foucault, P. Gounier (éd.), Paris, 1998, p. 486 ; la notice biographique de Charles Guérin assure que c'est Guérin qui a rédigé la partie du mémoire de Foucault relative à l'élection d'Avranches. La source de Foucault, qui s'est trompé sur les noms des évêques de Coutances et de Sées, n'est pas claire. 
mands de l'époque ducale ont choisi pour la consécration de leur église des dates qui n'étaient pas celle de leurs saints patrons, mais des dates importantes pour la communauté locale ${ }^{21}$; le seul saint dont la fête fut célébrée en Normandie le 17 septembre est Floscel (ou Floxel), un martyr du II $^{\mathrm{e}}$ siècle ${ }^{22}$. Notons également que le 18 septembre était la fête de saint Senier, un ancien évêque d'Avranches. Ses reliques appartenaient au trésor de l'ancienne cathédrale ${ }^{23}$, et il n'est pas impossible que Robert Cénalis ait simplement mal transcrit la date xiv calendas octobris (18 septembre) comme xv calendas octobris (17 septembre).

En outre, la notice biographique sur l'évêque Richard L'Évêque (1170-25 ou 26 avril 1182) nous livre avec un détail méconnu de l'histoire architecturale de la cathédrale. Cette notice concerne l'inhumation de l'évêque, dont le tombeau aurait été placé « autour de l'autel saint Martin, entre les piliers de l'église $»^{24}$. Cette dédicace en l'honneur du saint tourangeau est fort intéressante, car, selon Grégoire de Tours, l'évêque Léodovald qui dirigea l'église avranchinaise au $\mathrm{VI}^{\mathrm{e}}$ siècle, demanda des reliques de saint Martin pour sa cathédrale ${ }^{25}$. Pourtant, bien que les fouilles menées dans les années 1970 aient révélé l'existence d'un édifice antérieur à la cathédrale romane ayant pu les accueillir ${ }^{26}$, les reliques ne figurent pas dans les inventaires ${ }^{27}$, et furent vraisemblablement transférées à l'église Saint-Martin d'Avranches, qui existait encore au XIX ${ }^{\mathrm{e}}$ siècle, au sud de la ville. Il est néanmoins intéressant de constater qu'une trace d'une dévotion à saint Martin, l'un des premiers cultes promus par les évêques de Gaule, existait toujours dans la cathédrale au début du XVI ${ }^{\mathrm{e}}$ siècle.

C'est toutefois dans la notice sur l'évêque Guillaume I Burel (fin 1183/début 1184-17 ou 18 mars 1196 ${ }^{28}$ que l'intérêt du manuscrit Cénalis devient évidente. Cette notice est principalement composée d'extraits tirés d'un « ancien registre » (vetustus commentarius), peut-être l'inventaire de Laurent de Faye, qui nous

21 D. Bates, Odo, bishop of Bayeux, 1049-1097, thèse doctorale, University of Exeter, 1970, p. 47 ; M. LÉLÉGARD, «La cathédrale et la tombe de Geoffroi d'après le Livre noir de Coutances », dans Les évêques normands..., op. cit., p. 295-301 ; R. Allen, "The Acta archiepiscoporum... », op. cit., p. 17.

22 Taschenbuch der Zeitrechnung des Deutschen Mittelalters und der Neuzeit, 13e éd., H. Grotef end (éd.), Hanover, 1991, p. 55.

23 Son reliquaire fut brûlé par les protestants en 1562 : «Le lundy 8 mars 1562, l'eglise d'Avranches fut ravagee par les heretiques... la chasse d'argent ou etoient des reliques de Saint-Senier, qui avoit été mise en terre sous le chapitre, cinq autres chasses qui etoient deriere le chœur enterrees, deux autres chasses etant dans ledit pilier emportees et les saintes reliques brulees... ", Bibl. mun. Avranches, fonds Pigeon, ms. 45 , p. 487.

24 "Richardus, sepulchro conditus apud Arboricas, e regione altaris divi Martini, inter pilas ecclesię", BnF, ms. lat. 5201, fol. $77 \mathrm{v}$.

25 Grégoire de Tours, "Liber II de virtutibus s. Martini », B. Krusch (éd.), dans MGH, SS rer. Merov., 1.2, Hanovre, 1885, p. 622.

26 D. Levalet, « La cathédrale Saint-André... », op. cit., p. 112-116.

27 Ces inventaires sont édités dans, R. Allen, "Les reliques de la cathédrale d'Avranches ", Revue de l'Avranchin et du pays de Granville, 87, 2010, p. 501-535, aux p. 528-535.

28 Sur la mort de l'évêque, traditionnellement datée de 1194 (J. Peltzer, Canon law, careers and conquest..., op. cit., p. 155) voir la nouvelle édition de la soi-disant Chronique de Savigny, actuellement en cours de préparation par l'auteur. 
donne des renseignements précieux sur le testament que l'évêque Guillaume fit réaliser en faveur de son église. Parmi ces legs se trouvent les redevances de quelques possessions à Ponts ${ }^{29}$, perçues par les chanoines chaque 18 mars, jour anniversaire de la mort de l'évêque ${ }^{30}$, ainsi que la dîme d'un fief situé à Saint-Jean-de-la-Haize ${ }^{31}$, utilisée pour l'achat de l'huile de la lampe de l'autel Saint-Pierre ${ }^{32}$. L'évêque Guillaume donna également à la cathédrale plusieurs vêtements et vases sacrés, à savoir 8 pallia ; 3 tuniques ; 3 dalmatiques ; 3 aubes, dont une dorée ; 1 étole dorée ; 1 manipule doré ; 1 calice doré ; 2 petits vases en argent; 1 ciboire en argent, et 1 mitre et des sandales. Guillaume légua aussi de l'argent afin qu'un ange puisse être réalisé et déposé avec les ossements de saint André sur l'autel majeur pendant les jours solennels, ainsi que 2 encensoirs en argent, 1 navette à encens en argent avec sa petite cuillère, 1 chape de soie rouge, et 2 petits coussins, l'un de soie et l'autre de toile teinte avec le texte en fil d'argent ${ }^{33}$. Enfin, l'évêque versa 100 sous manceaux à la cathédrale pour la rédemption de ses ancêtres (ou de ses prédécesseurs $)^{34}$. Cet inventaire remarquable des ornements de la cathédrale à la fin du XII ${ }^{\mathrm{e}}$ siècle, déjà signalé mais jamais publiée ${ }^{35}$, est d'autant plus important que ce trésor ne resta pas longtemps dans l'église ; en effet, tous ces objets ont disparu de l'inventaire du trésor dressé un siècle plus $\operatorname{tard}^{36}$, et il est possible que plusieurs de ces articles précieux aient été emportés ou brûlés pendant le pillage d'Avranches, effectué en 1204 par Guy de Thouars, régent de Bretagne $(1203-1206)^{37}$.

29 Ponts, Manche, cant. Avranches.

30 Selon la Gallia christiana, la mort de Guillaume fut enregistrée le 18 octobre au nécrologe perdu de l'abbaye de Savigny, Gallia christiana, t. XI, col. 482.

31 Saint-Jean-de-la-Haize, Manche, cant. Avranches.

32 "Decimoquinto calendas aprilis obiit Gulielm(us) Burellus Abrincensis episcopus, qui legavit canonicis bujus ecclesię, qui eidem anniversario intererunt, redditum du[orum] sept[orum] de Pons, et ejusdem prati juxta adjacentis, et redditum unius masurę ibidem sitę. Legavit insuper decimam feodi Ham [a]', quod est in parochia sancti Joannis de Hesa, quam emerat ad oleum emendum in lampade coram altari beati Petri arsurum ", Paris, BnF, ms. lat. 5201, fol. 78v-79r.

33 «Legavit etiam ecclesię octo pallia, tres tunicas, tres dalmaticas, tres albas paratas, unam scilicet conparatam de aurato, stolam et manipulum deauratum, calicem intus et externis deauratum, duos urceolos argenteos, pyxidem argenteam ad reponendum hostias, mitram et sandalia episcopalia. Legavit etiam aurum et argentum ad faciendum angelum, qui sollemnibus diebusponitur super altare, cum reliquiis beatissimi Andree, et duo thuribula argentea, naviculam argenteam cum cocleari, capam etiam de samito rubeo, duas culcitras, unam sericeam, aliam de telis tinctis, et textum argenteum ", BnF, ms. lat. 5201, fol. 79r.

34 "Legavit et centum solidos Cenomanenses (sic) monetae, pro redemptione majorum », BnF, ms. lat. 5201, fol. 79 r. Le sens de la phrase «pro redemptione majorum» est peu clair. En latin classique, le mot "majores » peut se référer aux ancêtres, mais il est possible qu'en ce contexte il se réfère aux prédécesseurs de l'évêque. Pour un autre exemple de l'usage de cette phrase, voir Wirtembergisches urkundenbuch, Königliches Staatsarchiv in Stuttgart (éd.), Stuttgart, 11 vol., 1849-1913, t. II, no CCCIX, p. 5-6. Je tiens à remercier Richard Ashdowne, Kathrin Korn, Mark Thakkar et Carolinne White d'Oxford Dictionary of Medieval Latin from British Sources d'avoir proposé ces traductions et de m'avoir envoyé la référence ci-dessus.

35 Voir Gallia christiana, t. XI, col. 482 ; É.-A.Pigeon, Le diocèse d'Avranches..., op. cit., t. II, p. 330. 1295.

36 R. Allen, "Les reliques d'Avranches... ", op. cit., p. 528-530. L'inventaire fut rédigé en mai

37 Euvres de Rigord et de Guillaume le Breton, historiens de Philippe-Auguste, H.-F. Delaborde (éd.), Paris, 2 vol., 1882-1885, t. I, p. 221. 
La notice dédiée à Guillaume III Ptolomeus (fin 1199/début 1200-1210) est aussi importante que celle sur son prédécesseur. Elle commence, notamment, en faisant référence à une charte de Philippe Auguste (1180-1223), rédigée en 1209, la trentième année de son règne, par laquelle il octroya à la cathédrale cents livres de terre à Juilley ${ }^{38}$. Cette charte ne se trouve pas dans le recueil des actes du roi ${ }^{39}$, mais l'existence du document est corroborée par les notes du chanoine Guérin. Celles-ci précisent que la donation fut faite à Gisors en compensation des pertes subies par la cathédrale ${ }^{40}$, et qu'elle incluait des redevances prélevées sur les terres de Juilley, de Crollon ${ }^{41}$, de Vergoncey ${ }^{42}$, d'Angey $^{43}$, de Sainte-Pience ${ }^{44}$ et du fief du Homme, avec tout ce qu'Enjuger de Bohon ${ }^{45}$, Guillaume de Saint-Jean ${ }^{46}$ et Élie le bouteiller yavaient possédé ${ }^{47}$. Ces détails complémentaires sont très importants, d'une part parce que ces individus furent souvent à la cour Plantagenêt ${ }^{48}$, mais d'autre part du fait que nous pouvons démontrer qu'ils furent implantés sur ces terres ${ }^{49}$. Quant aux pertes subies par la cathédrale, l'abbé Pigeon les interprète comme les dommages infligés à l'église par Guy de Thouars ${ }^{50}$, alors qu'il est parfois admis que les reliques, soi-disant données à la cathédrale par saint Aubert, avaient été découvertes par Guillaume Ptolomeus à l'occasion des travaux de restauration de la cathédrale ${ }^{51}$, mais rien ne le prouve. La mention de cette charte royale

38 "Extat charta de centum libratis terre a rege Philippo concessis apud Julleyum, anno Domini millesimo ducentesimo nono, regni vero Philippici trigesimo ", BnF, ms. lat. 5201, fol. 79r. Juilley, Manche, cant. Ducey.

39 Aucun des six tomes n'en donne ni le texte, ni un extrait, ni une mention. Pour les actes de l'an 1209, voir Recueil des actes de Philippe Auguste, roi de France, H.-F. Delaborde et al. (éd.), Paris, 6 vol., 1916-2005, t. IV, nos $1060-1107$, p. 133-192.

40 Une charte semblable fut octroyée par le roi en faveur de la cathédrale de Coutances (datée d'octobre 1209, à Gisors), Le cartulaire du chapitre cathédral de Coutances. Étude et édition critique, J. FonTANEL (éd.), Saint-Lô, 2003, n 299, p. 439.

41 Crollon, Manche, cant. Ducey.

42 Vergoncey, Manche, cant. Saint-James.

43 Angey, Manche, cant. Sartilly.

44 Sainte-Pience, Manche, cant. La Haye-Pesnel.

45 Bohon, Manche, cant. Carentan.

46 Saint-Jean-le-Thomas, Manche, cant. Sartilly.

47 "Rex Franciae in recompensatione damnorum quae ecclesia Abrin(censis) [...] fuit, predicto rege dedit dictae ecclesiae et episcopis 100 l(i)b(ris) redditus a Juilley, Crollon, Vergoncé, Angé, Sancta Pientia et in feudo du Homme, cum omni integritate qua Angelg(er)us de Bouhon, Guill(elmus) de Sancto Jo(hann)e et Helias buticularius praedictas terras antea possederunt. Apud Gisorem 1209, et est litera signata signo regis ", Bibl. mun. Avranches, fonds Pigeon, ms. 45, p. 388. Malheureusement, cette note se trouve en haut de la page, où le papier est défraîchi et déchiré, ce qui rend au moins un mot illisible.

48 Pour Guillaume et Enjuger, voir Recueil des actes de Henri II, roi d'Angleterre et duc de Normandie, L. Delisle et E. Berger (éd.), Paris, 4 vol., 1916-1927, Introduction, p. 364-365, 500-501. Il semble que Élie le bouteiller soit précédemment inconnu.

49 L'église d'Angey, par exemple, fut donnée à l'abbaye de La Lucerne par Guillaume de Saint-Jean, Cartulaire de la Luzerne, F.-N. Dubosc (éd.), Saint-Lô, 1878, no VI, p. 4-6, à la p. 5. À propos d'Enjuger de Bohon et de ses possessions à Vergoncey et à Crollon, voir Recueil de jugements de l'Échiquier de Normandie au XIII siècle (1207-1270), L. Delisle (éd.), Paris, 1864, p. 32.

50 É.-A. Pigeon, Le diocèse d'Avranches..., op. cit., t. II, p. 331. Une charte de 1207 parle de l'état déplorable de la cathédrale : "... ecclesie nostre Abrinc(ensis) indigenciam attendentes, cujus fabrica ruinam multiplicem minabatur...", Bibl. mun. Avranches, ms. 206, fol. 94v.

51 D. Levalet, «La cathédrale Saint-André... », op. cit., p. 110. 
est néanmoins de première importance, non seulement du fait de son caractère totalement inédit, mais également parce qu'il s'agit de l'unique charte connue selon laquelle ce roi fit une donation à la cathédrale et aux évêques d'Avranches. Il est donc regrettable que Robert Cénalis et Charles Guérin aient omis de transcrire le texte complet de cet acte, qui devait être conservé dans les archives diocésaines ${ }^{52}$.

La deuxième partie de la notice sur Guillaume Ptolomeus mérite, en raison de son importance, d'être publiée en entier : ${ }^{53}$

"Undecimo calendas septembris obiit Gulielmus Ptolomei alias Pholonei, ex Rogerio patre et Heloide matre cujus anime propicietur Deus, ad cujus anniversarium solemniter faciendum, capitulum Abrincense tenetur annuatim solvere viginti solidos Cenomanenses de redditu de Valeriis. Idem vero episcopus terram de Valeriis, terram et redditus de Anglia, licet de antiquo jure ad Abrincensem ecclesiam spectantes, acquisivit, non sine magnis laboribus et expensis. Patronatus ecclesiarum videlicet de Milleyo, de la Godefrey, Sanctę Trinitatis de Nemore Randoni, alias Rantom, medietatem ecclesie Sancti Senerii acquisivit et capitulo Abrincensi, cum medietate ecclesie de Tania, in augmentum communie misericorditer contulit. Novum cimiterium, tam de deportationibus quam acquisitionibus, construxit. Platee vero domorum scilicet decani, Radulphi le Gerrier, Nicholai Chef de Bouc, Thome de Ponte, Roberti B[er]haudi, Richardi de Genesio, per eundem episcopum a dominis fundatorum fuerunt impetrate, et imperpetuum ecclesię Abrincensi concessę. Prębendam itaque de Tania constituit. Redditus proprios episcopatus, circumquaque viriliter dilatando, tam ex dono regis Francie quam alias multimode, augmentavit. Capas, vestimenta, pannos sericos, et alia ornamenta ecclesię Abrincensi contulit, non in hoc solum perficiens, sed in multis».

Le contenu de ce texte, tiré d'un cartulaire qui, comme nous le verrons, semble être le Livre Blanc, mentionne les noms des parents de l'évêque ; c'est là un détail tout à fait frappant. En effet, parmi les douze pontifes qui occupèrent l'évêché avant Guillaume, nous ne connaissons que le père et la mère de trois d'entre eux ${ }^{54}$. Le fait donc que nous proposions d'identifier ces individus avec Roger de Saint-André (fl. 1172) et sa femme, Héloïse (fl. 1181 - 1192),

52 Une note marginale effacée dans le manuscrit de Guérin lit comme suit : «[Ex regist.] ep(isco) patus ", Bibl. mun. Avranches, ms. 45, p. 388 (en marge). La Gallia christiana soutient que Guillaume " occurit ... anno 1209 in chartulario ecclesiae cathedralis ", qui est peut-être une référence à la charte de Philippe Auguste, Gallia christiana, t. XI, col. 484.

$53 \mathrm{BnF}$, ms. lat. 5201, fol. 79v-80r.

54 Il s'agit de Jean d'Ivry (1060-1067), de Richard de Subligny (1142/1143-1153) et de Guillaume I Burel. Voir, R. Allen, « "A proud and headstrong man" : John of Ivry, bishop of Avranches and archbishop of Rouen, 1060-79 », Historical Research, 83, 2010, p. 192 ; D. Power, The Norman frontier in the twelfth 
et que cette identification soit utile à la compréhension d'un événement capital de l'histoire du diocèse, augmente encore plus l'importance du manuscrit Cénalis.

L'élection de Guillaume Ptolomeus à l'épiscopat avait mis fin à une période troublée de l'histoire de l'évêché d'Avranches ${ }^{55}$. La mort de Guillaume I Burel avait marqué le début d'une longue vacance du siège épiscopal en raison du conflit consécutif à l'élection sur le siège épiscopal avranchais, en 1196, de Guillaume de Chemillé qui, un an plus tard, devint évêque d'Angers. Le pape Innocent III (1198-1216) avait été informé de cette translation par Nicolas de L'Aigle, écolâtre d'Avranches, et après une enquête sur l'affaire révélant que le chapitre avranchais avait élu Guillaume Ptolomeus comme nouvel évêque, le pape lui ordonna de recevoir ce dernier au siège épiscopal. Mais la nomination de Ptolomeus ne fut pas sans problèmes. Trois mois plus tard, le 17 mars 1199, le pape, ayant traité d'un autre appel sur cette affaire, invalida l'élection de Guillaume. Ce deuxième appel avait encore été lancé par Nicolas de L'Aigle, qui reprochait à Ptolomeus son illettrisme, ses origines extérieures au diocèse d'Avranches, ses liens étroits avec la cour royale, et enfin d'avoir plusieurs enfants ${ }^{56}$.

Certes, c'est grâce aux seules récriminations de son adversaire que nous connaissons ces détails sur l'évêque, mais, comme Jörg Peltzer l'a constaté, ces reproches sont si détaillés et si précis qu'ils ne peuvent être complètement rejetés $^{57}$. D'ailleurs, le manuscrit Cénalis confirme la deuxième accusation formulée par Nicolas. En effet, avant son élection au siège épiscopal, Guillaume Ptolomeus fut clerc de Guillaume fils Raoul, sénéchal de Normandie. Au cours de ces années, ses activités apparaissent principalement dans la documentation historique relative aux institutions correspondant à l'actuel département de 1 'Eure ${ }^{58}$. C'est précisément en cette partie de la Normandie, à une époque coïncidant du point de vue chronologique, que nous rencontrons un couple formé par un dénommé Roger de Saint-André et sa femme, Héloïse ${ }^{59}$. Sans lien véritablement avéré, bien sûr, une telle parenté reste hypothétique. Par

and early thirteenth centuries, Cambridge, 2004, p. 518 ; J. Peltzer, Canon law, careers and conquest..., op. cit., p. 155 , n. 382.

55 Pour ce qui suit, voir J. Peltzzer, «Portchester, les évêques d'Avranches... », op. cit., p. 469-471; J. Peltzer, Canon law, careers and conquest..., op. cit., p. 155-164.

56 «... qui propter ignorantiam litterarum et quia de gremio non erat ecclesie, nisi ejus clericis antea reprobatis, non poterat postulari... ", Die Register Innocenz'III, O. HAgeneder et al. (éd.), Rome, 10 vol., 1964-, t. II, $n^{\circ}$ 18. Un différend entre Nicolas de L'Aigle, devenu doyen d'Avranches (1211-1223), et Geoffroy de la Champagne, fut résolu devant «le fils du seigneur Guillaume, évêque d'Avranches » (filio domini Willelmi episcopi Abrincensis), Bibl. mun. Avranches, ms. 206, fol. 16v. Il est possible qu'il s'agisse du fils de Guillaume Ptolomeus.

57 J. Peltzer, Canon law, careers and conquest..., op. cit., p. 162.

58 Ibid., p. 162, n. 414.

59 D. Power, The Norman frontier..., op. cit., p. 483. Saint-André-en-la-Marche, maintenant SaintAndré-de-1'Eure. Eure, ch.-1. cant. 
ailleurs, nous savons que Guillaume fut actif dans les environs de l'Avranchin avant même son élection, puisqu'il paya à Richard de Fleury dix livres afin qu'il soit reconnu comme son héritier ${ }^{60}$. La mention, désormais publiée, des parents de Guillaume dans le manuscrit Cénalis permet à présent d'espérer prochainement une identification définitive.

En plus de ces renseignements généalogiques, l'extrait ci-dessus présente de nombreuses informations méconnues sur les activités temporelles de l'évêque Guillaume. Comme son prédécesseur, il fit son testament au profit du chapitre cathédral, qui reçut, chaque année le 22 août, 20 sous manceaux de la redevance prélevée sur la terre de Vallière ${ }^{61}$. L'évêque assura également la possession de cette même terre, ainsi que les terres et les revenus anglais qui furent dus à la cathédrale selon "le droit ancien " (de antiquo jure). Il s'agit, dans ce dernier cas, des terres de Portchester (Hampshire), que Guillaume avait récupérées de Guillaume du Hommet, connétable du roi, qui les avait tenues de l'évêque, mais pour lesquelles il n'avait pas payé la ferme pendant douze ans. Cette référence aux efforts de l'évêque Guillaume prouve que Robert Cénalis avait consulté le Livre Blanc; en effet, bien qu'il y ait des chartes dans le Livre Vert au sujet des terres anglaises ${ }^{62}$, le seul acte connu de Guillaume Ptolomeus concernant Portchester se trouvait autrefois dans ce cartulaire perdu ${ }^{63}$.

La consultation du Livre Blanc par Cénalis est également confirmée par la liste des églises pour lesquelles Guillaume aurait obtenu le droit de patronage. Par exemple, selon une autre partie de la notice biographique sur Ptolomeus, cet évêque aurait reçu de Roger de Milly le droit de patronage de l'église de Milly ${ }^{64}$, la charte traitant de ce don se trouvant dans un cartulaire ${ }^{65}$. Il existe une charte dans le Livre Vert concernant l'église de Milly, mais ce document dit qu'un certain Pierre Rastellus avait résigné l'église entre les mains d'un évêque nommé Guillaume ${ }^{66}$, peut-être Ptolomeus, mais plus probablement l'un de ses successeurs. Il n'y a également aucune charte dans le Livre Vert concernant l'évêque Guillaume et le droit de patronage des églises de La Godefroy et de

60 Magni rotuli scaccarii Normannice sub regibus Anglice, T. Sta Pleton (éd.), Londres, 2 vol., 18401844, t. II, p. 295. Fleury, Manche, cant. Villedieu-les-Poêles.

61 Vallière, Manche, cant. Saint-Malo-de-la-Lande. Cette terre entra dans le temporel de la cathédrale au XI ${ }^{\mathrm{e}}$ siècle, R. AlLEN, "Un évêque et sa ville ... », op. cit., p. 41.

62 Bibl. mun. Avranches, ms. 206, fol. 6v-7v.

63 Cette charte est éditée dans J. Peltzer, "Portchester, les évêques d'Avranches ... ", op. cit., p. 481-482.

64 Un Roger de Milly se trouve parmi les témoins d'une charte de Roland, doyen du chapitre d'Avranches (1161-11 nov. 1177, mais peut-être jusqu'en 1187/8), Bibl. mun. Avranches, ms. 206, fol. $32 \mathrm{r}-\mathrm{v}$.

65 «Hic [Guillelmus episcopus] etiam a Rogerio de Milleyo milite recepit jus patronatus ecclesię de Milleyo: in eam rem confectum est diploma in chartulario ", $\mathrm{BnF}$, ms. lat. 5201, fol. 79r.

66 Bibl. mun. Avranches, ms. 206, fol. 3v-4r. Cette charte est non datée. Milly, Manche, cant. Saint-Hilaire-du-Harcouët. 
Ronthon ${ }^{67}$, qui selon ce même cartulaire furent respectivement données à la cathédrale par l'évêque Richard de Subligny et un évêque Guillaume ${ }^{68}$, tandis que deux chartes du Livre Vert concernent l'église de Saint-Senier (peut-être Saint-Sénier-de-Beuvron, ou bien Saint-Sénier-sous-Avranches), la première étant une contestation de Geoffroy de la Champagne et la seconde une confirmation de Geoffroy de Saint-Senier ${ }^{69}$. Enfin, des deux chartes trouvées dans le Livre Vert qui traitent de l'église de Tanis ${ }^{70}$, 1'une est seulement signalée par l'inventaire au début du manuscrit, les feuillets sur lesquels elle fut écrite ayant disparu $^{71}$, et l'autre est une bulle du pape Honorius III (1216-1227) ${ }^{72}$.

Cet aperçu remarquable des activités jusqu'à lors ignorées de Guillaume est bien loin de l'image du prélat véhiculée par les accusations de Nicolas de L'Aigle. Selon l'extrait précité, l'acquisition des droits de patronage n'était qu'une petite partie des contributions faites par l'évêque à son église. En effet, il aménagea un nouveau cimetière pour la cathédrale ${ }^{73}$, et obtint les emplacements des maisons du doyen et de divers autres personnages, qu'il donna au chapitre. Parce que le cartulaire dit Livre Vert ne contient aucun renseignement sur ces acquisitions, il semble évident que cette information est tirée du Livre Blanc; et, bien que ces chartes n'existent plus, les personnages cités par Cénalis peuvent être associés à l'évêque. Raoul le Gerrier, par exemple, se trouve parmi les témoins d'une charte de Guillaume Ptolomeus où il figure en tant qu'official de l'évêque ${ }^{74}$, tandis que Robert Berhaut peut être non seulement identifié parmi les témoins des actes de l'évêque ${ }^{75}$, mais il fut également son scribe $^{76}$. Le Livore Vert confirme même qu'il possédait des terres à Avranches ${ }^{77}$. Nicolas Chef de Bouc (Tête de Bouc) apparaît dans ce même cartulaire avec le titre d'écolâtre, et fit plusieurs donations à la cathédrale ${ }^{78}$. Un maître Richard de Genêts, chantre de la cathédrale, est témoin d'une charte de janvier 1231, où l'on trouve aussi un maître Robert Berhaut, qualifié d'archidiacre ${ }^{79}$. Thomas

67 La Godefroy, Manche, cant. Avranches; Ronthon (maintenant Dragey-Ronthon), Manche, cant. Sartilly.

68 Bibl. mun. Avranches, ms. 206, fol. 2v et 4r-v.

69 Bibl. mun. Avranches, ms. 206, fol. 3v et 17v-18r. Les églises de Saint-Sénier-de-Beuvron (Manche, cant. Saint-James) et Saint-Sénier-sous-Avranches (Manche, cant. Avranches) entrèrent dans le temporel de la cathédrale au XI ${ }^{\mathrm{e}}$ siècle, R. Allen, «Un évêque et sa ville ... », op. cit., p. 40-41.

70 Tanis, Manche, cant. Pontorson.

71 «[P] rivileg(ium) Gregorii pape super patronatu de Tania», Bibl. mun. Avranches, ms. 206, fol. 1r. Le pape ne peut pas être identifié avec certitude.

72 Bibl. mun. Avranches, ms. 206, fol. 3r. Cette charte est datée du 2 avril 1224.

73 D. Levalet, «La cathédrale Saint-André ...», op. cit., p. 123.

74 Arch. nat., L 976, nº 1091.

75 Arch. nat., L 974, $\mathrm{n}^{\circ} 901$.

76 Arch. nat., L 976, $\mathrm{n}^{\circ} 1091$.

77 Bibl. mun. Avranches, ms. 206, fol. 10v-11r. Cette terre fut située à côté de la maison du doyen (juxta domum decani).

78 Nicolas, dont le surnom est aussi écrit "Chif de Boc ", "Chief de Bouc » et "Chief de Boc », voulut que le chapitre lui fît un service anniversaire, et à cet effet lui donna une vigne et une masure avec les édifices bâtis dessus, Bibl. mun. Avranches, ms. 206, fol. 14r-v.

79 Arch. nat., L 975, n 1059. 
de Ponts est malheureusement inconnu, mais un certain Robert de Ponts se trouve dans une charte d'un évêque Guillaume, où il est qualifié de chantre de la cathédrale ${ }^{80}$. Selon le manuscrit Cénalis, l'évêque fonda également la prébende de Tanis, qui au $\mathrm{Xv}^{\mathrm{e}}$ siècle, avait un revenu de 15 livres ${ }^{81}$, tandis qu'il avait augmenté les redevances de son évêché " par le don du roi ainsi que diverses autres donations ». Comme son prédécesseur, Guillaume Ptolomeus avait aussi donné beaucoup d'ornements à sa cathédrale, y compris des chapes, des vêtements et des tissus de soie, qui remplacèrent peut-être les objets détruits lors du pillage de 1204.

La mort de Guillaume Ptolomeus, en 1210, ne provoqua pas de controverse semblable à celle qui eut lieu après le décès de son prédécesseur. Le nouvel évêque, Guillaume IV Burel (1210-1236), connu aussi comme de Ostilleio ${ }^{82}$, avait auparavant été chanoine de la cathédrale du Mans ${ }^{83}$. Malheureusement, le manuscrit Cénalis nous fournit peu de renseignements sur son épiscopat. Cependant, la notice qui lui est consacrée contient deux informations intéressantes.

La première concerne les legs de l'évêque, dont les détails furent trouvés dans le Livre Blanc. Selon ce manuscrit, l'évêque Guillaume ordonna que le jour anniversaire de sa mort ${ }^{84}$, qui survint un 29 octobre, le chapitre cathédral devait recevoir 50 sous et cinq quartiers de froment de la terre de Ronthon ${ }^{85}$. La seconde information concerne d'Alexandre de Villedieu ${ }^{86}$, grammairien bien connu dont les livres pédagogiques furent longtemps utilisés dans toutes les écoles au Moyen Âge ${ }^{87}$. D'après le Livre Blanc, cet auteur du XIII ${ }^{\mathrm{e}}$ siècle avait été chanoine de la cathédrale d'Avranches, et sa mort y fut commémorée le 29 juillet $^{88}$. La source du lien entre Alexandre et la cathédrale d'Avranches, bien connue des érudits du XIX ${ }^{\mathrm{e}}$ siècle $^{89}$, est souvent ignorée par leurs successeurs

80 "Pro magistro Rob(er)to de Po(n)te quondam cantore.X. s(olidos) Tur(onensis)», Bibl. mun. Avranches, ms. 206, fol. 10v.

81 Pouillés de la province de Rouen, A. Longnon (éd.), Paris, 1903, p. 160.

82 Peut-être Outillé, Sarthe, cant. Écommoy, cne. Saint-Mars-d'Outillé, J. Peltzer, Canon law, careers and conquest..., op. cit., p. 165 , n. 427.

83 J. Peltzer, Canon law, careers and conquest..., op. cit., p. 165, n. 427.

84 Selon la chronique de Savigny et l'obituaire de l'abbaye de La Lucerne, l'évêque Guillaume est décédé le 28 octobre, Miscellaneorum libri septem, hoc est, collectio veterum monumentorum, E. BALuze (éd.), Paris, 7 vol., 1678-1715, t. II, p. 319 ; A. Du Monstier, Neustria pia, Rouen, 1663, p. 797.

85 La charte à laquelle se réfère cette notice se trouve dans: « Ex chartulario : Quarto calendas novembris obiit bone memorię Gulielmus de Ostilleyo episcopus Abrincensis, cujus obitu babent canonici quinquaginta solidos percipiendos in communia sua, per manum communiarii : et pro illis quinquaginta solidis, canonici habent quinque quarteria frumenti que episcopus dederat eis, ad anniversarium faciendum apud Ronton ", $\mathrm{BnF}, \mathrm{ms}$. lat. 5201, fol. 80v-81r. Bibl. mun. Avranches, ms. 206, fol. 4r-v.

86 Villedieu-les-Poêles, Manche, chef-lieu.

87 Pour des renseignements biographiques et bibliographiques, voir P. Glorieux, La faculté des arts et ses maitres au XIII siècle, Paris, 1971, p. 86-88; Dictionnaire des philosophes médiévaux, B. PATAR (éd.), Saint-Laurent, 2006, p. 476-477.

88 "Quod ne quis diffidat, id ex chartulario perspicuum erit, in quo ita legitur: Quarto calendas augusti obiit Alexa(n) der de Villa Dei canonicus sancti Andree, in cujus obitu canonici percipiunt solidos Turonicos decem", BnF, ms. lat. 5201, fol. 81r.

89 A. M. Laisné, " Notice bibliographique sur Alexandre de Villedieu », Mémoires de la Société d'archéologie d'Avranches, 2, 1859, p. 89 ; E.A. Pigeon, Le diocèse d'Avranches..., op. cit., t. II, p. 483 n. 
modernes ${ }^{90}$. Les dates de la présence d'Alexandre à Avranches sont inconnues. Selon un manuscrit de son principal ouvrage, le Doctrinale puerorum, le jeune Alexandre fut étudiant à Paris, et avait commencé ses activités pédagogiques à Villedieu avant d'être sollicité par l'évêque de Dol afin d'instruire ses neveux ${ }^{91}$. Sa seule apparition dans une source avranchaise, en dehors du Livre Blanc, se trouve dans le Livre Vert, où un certain Alexandre, chanoine et maître, donne à la cathédrale un emplacement avec son édifice, qu'il tient de Jean de La Mouche ${ }^{92}$, chevalier. Cet emplacement se trouvait à proximité de la porte de la cathédrale, entre le mur du roi et celui de Nicolas l'orfèvre ${ }^{93}$. Si ce mur royal se réfère aux fortifications érigées par Louis IX en 1231, nous avons ici un terminus a quo pour l'arrivée d'Alexandre au chapitre cathédral ${ }^{94}$. Ceci correspond à ce que nous connaissons de Jean de La Mouche, qui selon une liste des noms de certains hommes illustres tirés des documents de l'abbaye de La Lucerne, était toujours en vie en $1232^{95}$.

La dernière note que nous considérerons ici, est consacrée à Guillaume de Sainte-Mère-Église (1236-1253) et se compose principalement de renseignements sur le roi Louis IX. En effet, hormis une note cryptique sur quelques acquisitions faites par l'évêque à Caen ${ }^{96}$, qui est répétée par la Gallia christiana ${ }^{97}$, mais dont aucune trace ne peut plus être trouvée, il n'y a que deux informations sur l'épiscopat de ce pontife. La première concerne le tombeau de l'évêque, qui se trouvait « autour de l'autel majeur, à gauche $»^{98}$. Cette affirmation contredit à première vue la Gallia christiana, qui soutient que l'évêque fut enterré dans

90 F. J. E. Raby, A history of secular Latin poetry in the Middle Ages, 2e éd., Oxford, 2 vol., 1957, t. II, p. 86-88.

91 P. J. Ford, «Alexandre de Villedieu's Doctrinale puerorum : a medieval bestseller and its fortune in the Renaissance ", dans G. H. Tucker (dir.), Forms of the "medieval" in the "Renaissance": a multidisciplinary exploration of a cultural continuum, Charlottesville, VA, 2000, p. 155 et n. 2.

92 La Mouche, Manche, cant. La Haye-Pesnel.

93 «... factum est quod magister Alexand(er) canonicus Abr(incensis) plateam suam cum edificiis quam de me tenebat dedit ecclesie Beati Andree in perpetuam et liberam elemosinam ... Dicta autem platea est justa (sic) portam ecclesie Sancti Andree inter murum domini regis et murum Nicholai aurifabri ... ", Bibl. mun. Avranches, ms. 206, fol. 18v.

94 Veterum scriptorum et monumentorum bistoricorum, dogmaticorum, moralium, amplissima collectio, E. Martène et U. Durand (éd.), Paris, 9 vol., 1724-1733, t. I, col. 1254. Robert Cénalis dit qu'il a trouvé le texte de cet acte dans un cartulaire ( $i d$ constat ex chartulario », BnF, ms. lat. 5201, fol. 80v), mais puisqu'une copie de cette charte se trouve dans un cartulaire de Philippe Auguste (Arch. nat., JJ 26, fol. VII $\left.{ }^{\mathrm{xx}} \mathrm{I}\right)$, on ne sait pas exactement s'il a consulté ce manuscrit ou un cartulaire perdu de la cathédrale d'Avranches. Heureusement, une note du chanoine Guérin confirme que le texte de cet acte fut autrefois trouvé dans l'inventaire de Laurent de Faye : « Ex regist. Rex coepit jardinum episcopi, in quo fieri fecit fossata et fortalitium suum et in recompensationem dedit 12 li(bras) redd(itus) super praeposituram de Abrin(censi), mense novembri 1231 », Bibl. mun. Avranches, fonds Pigeon, ms. 45, p. 390 (en marge). Il y a une charte de juillet 1269 dans le Livre Vert dans laquelle Louis parle des fortifications d'Avranches comme « nos murs» (muros nostre), Bibl. mun. Avranches, ms. 206, fol. 92v.

95 BnF, ms. fr. 4902, fol. 285r.

96 "Nonnulla acquisivit Cadomi, anno Domini millesimo ducentesimo quinquagesimo, ut ex chartulario diplomata probant ", BnF, ms. lat. 5201, fol. 81r.

97 Gallia christiana, t. XI, col. 485.

98 "Sepelitur Abrincis, e regione altaris majoris, ad sinistram», BnF, ms. lat. 5201, fol. 81r. 
la chapelle Notre-Dame, à gauche de l'autel ${ }^{99}$, tandis qu'un plan terrier de la cathédrale, recopié par l'abbé Pigeon d'après « les dessins de M. Lefebvre, ingénieur en chef ... de la généralité de Caen », et qui date de 1786, montre aussi le tombeau de Guillaume à cet endroit ${ }^{100}$. Ce même plan signale le tombeau de Guillaume Ir Burel à la gauche de l'autel majeur, alors que selon le manuscrit Cénalis et la Gallia christiana, cet évêque aurait été enterré à côté de la porte du vestiaire ${ }^{101}$. L'abbé Pigeon d'autre part soutient que le tombeau de Guillaume de Sainte-Mère-Église fut trouvé « au haut du chœur et au bas du sanctuaire $~^{102}$. Il est difficile de résoudre cette confusion quant à l'emplacement de ce tombeau puisque la cathédrale n'existe plus, mais nous accordons le bénéfice du doute au témoignage de Robert Cénalis, qui connaissait mieux l'édifice que les autres érudits déjà cités. En effet, l'abbé Pigeon fit souvent preuve de beaucoup d'imagination en décrivant l'histoire architecturale de cette église ${ }^{103}$, dont la dernière trace est rasée pendant son enfance ${ }^{104}$.

La deuxième note concerne l'un des legs faits à la cathédrale par Guillaume de Sainte-Mère-Église. Selon le manuscrit Cénalis, il y avait sur l'autel majeur de la cathédrale un cercueil en argent sur lequel une inscription précisait que l'évêque Guillaume l'avait fait recouvrir de lames d'argent ${ }^{105}$. Identifié par l'abbé Pigeon comme le reliquaire renfermant les restes donnés par saint Aubert ${ }^{106}$, il est plus probable que cette inscription se trouvait en fait sur la châsse contenant les reliques de saint André, qui occupaient une place de première importance dans cette partie de l'église. Le relevé de cette inscription est fautif puisque cette dernière affirme que l'évêque avait fait recouvrir le reliquaire en l'an 1257 ; or, à cette date, il était déjà mort depuis quatre ans. Il est cependant possible que Cénalis ait simplement mal transcrit septimo pour secundo, et même s'il a bien enregistré la teneur de cette épigraphe, il ne fait aucun doute que cet objet peu connu fut autrefois trouvé dans la cathédrale.

Tous ces renseignements révèlent l'importance du manuscrit Cénalis pour les spécialistes de l'histoire médiévale d'Avranches. Les informations qu'il

99 Gallia christiana, t. XI, col. 485.

100 Bibl. mun. Avranches, fonds Pigeon, ms. CP 9, fol. 105v.

101 «Sepultura donatus est apud Abrincas in ipso vestibuli hostio», BnF, ms. lat. 5201, fol. 78v; « Sepultus est Abrincis in ipso cathedralis ecclesia vestibuli ostio ", Gallia christiana, t. XI, col. 482.

102 É.-A. Pigeon, Le diocèse d'Avranches..., op. cit., t. II, p. 686.

103 D. Levalet, « La cathédrale Saint-André... », op. cit., p. 110.

104 É.-A. Pigeon, Le diocèse d'Avranches..., op. cit., II, p. 687-693.

105 «De hoc Gulielmo hęc legitur inscriptis, in conditorio argenteo, quod majori altari supereminet: "Anno Domini millesimo ducentesimo quinquagesimo septimo (sic), factum est latus hujus capsule, de argento et pecunia bonę memorię Gulielmi de Saincte (sic) Marie, ecclesię quondam episcopi Abrincensis" ", BnF, ms. lat. 5201, fol. 81r-v.

106 É.-A. Pigeon, Le diocèse d'Avranches..., op. cit., t. II, p. 332. 
contient nous permettent de réexaminer plusieurs aspects de la vie épiscopale avranchaise, et plus largement des aspects inédits de l'histoire anglonormande.

Cependant, même pour un diocèse qui souffre aujourd'hui cruellement de la disparition de ses documents médiévaux, l'importance du manuscrit Cénalis ne réside pas seulement dans sa valeur factuelle. En effet, avec des références et extraits tirés d'autres manuscrits, on redécouvre peu à peu le Livre Blanc, ce cartulaire aujourd'hui perdu de la bibliothèque cathédrale médiévale. Grâce aux notes du chanoine Guérin, nous connaissions déjà quelques chartes ainsi que des éléments du pouillé qui furent copiés d'après le cartulaire disparu. Avec les extraits contenus dans le manuscrit de Robert Cénalis, il apparaît que le Livre Blanc contenait également des notes nécrologiques, dans lesquelles furent résumés les dons faits par certains personnages à la cathédrale. Au milieu de ces notes figurent bien entendu les évêques d'Avranches mais aussi des personnalités comme Alexandre de Villedieu, qui souhaita que le chapitre lui fit un service anniversaire, et qui, à cet effet légua une somme annuelle de dix sous de tournois. Certes, faute de références suffisantes, il est toujours possible que les éléments transcrits par Cénalis aient été extraits de l'inventaire rédigé par Laurent de Faye, voire d'un autre manuscrit inconnu ; et, bien entendu, des références directes à la foliotation du Livre Blanc auraient permis une " reconstitution codicologique » nettement plus aisée. Néanmoins, grâce au manuscrit Cénalis, il est non seulement envisageable de restituer une partie de l'histoire oublie de cet ancien diocèse, mais également l'un de ses trésors documentaires disparu depuis deux siècles ${ }^{107}$.

107 L'auteur est en train de préparer un « essai de reconstitution » du Livre Blanc ainsi que de l'Inventaire de Laurent de Faye, pour lesquels environ 44 documents, complets ou fragmentaires, ont jusqu'à présent été retrouvés. 
Annexe : L'enterrement des évêques d'Avranches, ca. 1022-1253, selon le manuscrit Cénalis

\begin{tabular}{|c|c|c|c|}
\hline Évêque & Date de décès & Enterré & Feuillet \\
\hline Maugis & ca. 1022 - ca. 1026 & $\begin{array}{l}\text { «... in fundamentis turris majoris } \\
\text { campanae septentrionem versus » }\end{array}$ & 37 \\
\hline Hugues & ca. 1055 - ca. 1060 & aucun renseignement ${ }^{1}$ & -- \\
\hline Jean d'Ivry & 9 septembre 1079 & aucun renseignement ${ }^{2}$ & -- \\
\hline Michel & 26 janvier 1094 & $\begin{array}{l}\text { "Sepultus Michael quiescit in ec- } \\
\text { clesia Abrincensi e regione ejusdem } \\
\text { altaris occidentem versus ..." }\end{array}$ & $57 \mathrm{v}$ \\
\hline Turgis & 7 janvier 1134 & $\begin{array}{l}\text { "Turgisius e regione altaris Beate } \\
\text { Marie sepultus ad septentrionem }\end{array}$ & $61 \mathrm{r}$ \\
\hline $\begin{array}{l}\text { Richard de } \\
\text { Beaufou }\end{array}$ & 25 avril 1142 & $\begin{array}{l}\text { "Richardus de Bellafago apud } \\
\text { Beccum sepulchro conditus...» }\end{array}$ & $69 \mathrm{r}$ \\
\hline $\begin{array}{l}\text { Richard de } \\
\text { Subligny }\end{array}$ & $\begin{array}{l}18 \text { ou } 19 \text { juillet } \\
1153\end{array}$ & $\begin{array}{l}\text { "Richardus de Sullygneio apud } \\
\text { Sequellammelle sepultus... " }{ }^{4}\end{array}$ & $65 \mathrm{r}$ \\
\hline Herbert & 6 septembre 1161 & $\begin{array}{l}\text { "... sepultus in ęde Beatę Marie } \\
\text { Becci ....» }\end{array}$ & $70 v$ \\
\hline Achard & $\begin{array}{l}29 \text { ou } 30 \text { mars } \\
1170\end{array}$ & $\begin{array}{l}\text { «Sepultus est in templo semper } \\
\text { et superbeatę et perpetuo } \\
\text { benedictę Trinitatis de Lucerna, } \\
\text { Pręmonstratensis ordinis » }\end{array}$ & $77 \mathrm{v}$ \\
\hline Richard L'Évêque & 25 ou 26 avril 1182 & $\begin{array}{l}\text { "Richardus, sepulchro conditus } \\
\text { apud Arboricas, e regione altaris } \\
\text { divi Martini, inter pilas ecclesię" }\end{array}$ & $77 \mathrm{v}$ \\
\hline Guillaume I Burel & 17 ou 18 mars 1196 & $\begin{array}{l}\text { "Sepultura donatus est apud } \\
\text { Abrincas in ipso vestibuli hostio » }\end{array}$ & $78 v$ \\
\hline $\begin{array}{l}\text { Guillaume II } \\
\text { Ptolomeus }\end{array}$ & 22 août 1210 & $\begin{array}{l}\text { "Gulielmus Pholonei, ante } \\
\text { majus altare sepultus ad partem } \\
\text { dexteram » }\end{array}$ & 79 \\
\hline $\begin{array}{l}\text { Guillaume IV } \\
\text { Burel }\end{array}$ & 28 octobre 1236 & $\begin{array}{l}\text { "Gulielmus de Ostilleyo sepultus } \\
\text { ad Savigniac(e)n(sem) e regione } \\
\text { pręcipui altaris ...» }\end{array}$ & $80 r-v$ \\
\hline $\begin{array}{l}\text { Guillaume V de } \\
\text { Sainte-Mère-Église }\end{array}$ & $\begin{array}{l}17 \text { ou } 18 \text { février } \\
1253\end{array}$ & $\begin{array}{l}\text { "Sepelitur Abrincis, e regione } \\
\text { altaris maioris, ad sinistram ..." }\end{array}$ & $81 \mathrm{r}$ \\
\hline
\end{tabular}

1 La Gallia christiana soutient que l'évêque Hugues fut inhumé dans la cathédrale, Gallia christiana, t. XI, col. 475. Le plan terrier de M. Lefèbvre montre le tombeau d'Hugues à côté de ceux de Maugis et de Michel, Bibl.mun. Avranches, fonds Pigeon, ms. CP 9, fol. 105v.

2 Jean fut inhumé dans le baptistère de la cathédrale de Rouen, Orderic Vital, M. Chibnall (éd.), t. III, p. 22.

3 La note suivante se trouve dans un manuscrit des fonds Pigeon : «Hic Michaelis sepulturae locus videtur confirmare quod super edificationis ecclesiae Abrincensis tempore notavi quia nullius episcoporum praecedentium sepultura noscitur in ecclesia Abrincensi ante Michaelem, qui primus in ea conditus, et predictus locus sepulturae in infima parte sub turre scilicet innuit non usum sepeliendi in ecclesia vel saltem quod ab initio non in eminenti sed humili loco illustres mortui tantummodo sepeliebantur in ecclesiis ... », Bibl.mun. Avranches, fonds Pigeon, ms. 45, p. 162. Selon l'abbé Pigeon, il avait trouvé une autre note, que je n'ai pas pu retrouver, dans ce même manuscrit qui dit que Michel fut enterré «sub turre magnarum campanarum a parte septentionali ", É.-A.Pigeon, Le diocèse d'Avranches..., op. cit., t. II, p. 679.

4 Ce lieu n'a pu être identifié, mais il est probablement à rechercher en Italie où Richard, revenant de Rome, fut capturé par des brigands, M. Arnoux, Des clercs au service de la réforme : études et documents sur les chanoines réguliers de la province de Rouen, Turnhout, 2000, p. 279-280. 


\title{
Résumé
}

Robert Cénalis et l'histoire épiscopale d'Avranches de 1100 à 1253. - En raison de l'effondrement de la cathédrale d'Avranches au crépuscule de l'Ancien Régime et de la destruction des Archives départementales de la Manche en 1944, l'histoire du diocèse d'Avranches est très mal servie par la documentation médiévale. Il nous reste cependant quelques collections d'érudits contenant des copies de documents avranchais dont l'une des plus importantes fut compilée par Robert Cénalis, évêque d'Avranches (1532-1560). Conservé actuellement à la Bibliothèque nationale de France, ce manuscrit méconnu, dont le contenu est souvent tiré de documents perdus depuis longtemps, nous offre un aperçu important sur l'histoire du diocèse à partir du début du XII ${ }^{\mathrm{e}}$ siècle jusqu'au milieu du XIII ${ }^{\mathrm{e}}$, ainsi que la possibilité de mieux nous renseigner sur plusieurs faits diplomatiques, archéologiques, prosopographiques et topographiques.

Mots clés : Avranches, Robert Cénalis, Hierarchia Neustria, évêques, cathédrale, cartulaire, Livre blanc.

\begin{abstract}
Robert Cénalis and the episcopal history of Avranches, 1100-1253. - The collapse of the cathedral of Avranches towards the end of the Ancien Régime and the destruction of the departmental archives of La Manche in 1944 mean that the history of the diocese of Avranches is very poorly served by medieval documentation. There remain, however, certain antiquarian collections that do contain copies of Avranches material, of which one of the most important was compiled by Robert Cénalis, bishop of Avranches (1532-1560). Currently conserved at the Bibliothèque nationale de France, this little-known manuscript, whose contents is often taken from documents now lost, offers an important insight into the history of the diocese from the beginning of the twelfth century through to the mid-thirteenth, as well as the opportunity to better understand various diplomatic, archaeological, prosopographical and topographical facts.
\end{abstract}

Keywords : Avranches, Robert Cénalis, Hierarchia Neustria, bishops, cathedral, cartulary, Livre blanc. 